\section{How Should Research Be Funded? Difficulties With the Argument for Proportionality to Causes of Death or Years of Life Lost}

\author{
Vinay Prasad, MD, MPH
}

Whether research funding and charitable donations correlate with the major causes of cancer death is increasingly discussed and criticized in the popular press. ${ }^{1-4}$ For example, one news article notes that breast cancer receives more research funding than lung cancer, although the latter is responsible for more deaths. ${ }^{1}$ A slight modification to this argument is found in academic journals, in which authors lament the fact that funding for different tumor types or different diseases is not proportionate to years of life lost or disability-adjusted life years lost. ${ }^{5,6}$ Although these criticisms are initially compelling, on closer reflection they are flawed. Although it is plausible that disease funding and donations should be proportionate to some measure of the harm or damage done by a particular disease or specific tumor type, it is not certain that this will in turn result in the greatest benefit. Making this assumption is a straightforward logical error. Research funding ought to maximize the potential absolute risk reduction from research gains, and need not be strictly proportionate to measures of the severity of disease.

Consider a general and incontrovertible principle of ethical funding, which many tacitly embrace but which is rarely stated outright: the optimal funding portfolio will decrease the burden of disease as greatly as possible. This is the unspoken principle that underlies much of the discussion of funding. Put another way: the most logical and ethical way to fund cancer research by tumor type would be to do so in a way that returns the most life years to our patients. This is what philosophers call the utilitarian answer. All things being equal, we should fund projects that will give us the most years of quality life back.

Now consider the first approximation to this question, embodied by articles that contrast causes of death with amounts of donation or funding. These are all based in the premise that funding should be proportionate to what robs us of life. But, although it may be reasonable to think that such a funding system may do the most good, we must acknowledge that it is mistaken, for the simple reason that causes of death vary by age. For this reason, more infrequent causes of death may actually result in larger numbers of life years lost. For example, if cancer strikes down more people in the prime of life, while heart disease is responsible for more deaths in later years, cancer may be a more important public health problem.

It turns out that this is the case. Years of life lost-a measure that takes into account the untimeliness of death-is bigger for cancer than for heart disease. ${ }^{7}$ Perhaps this explains why, intuitively, the public may donate more to cancer than heart disease. ${ }^{2}$

And yet, even years of life lost (adjusted or not adjusted for disability) remains an imperfect proxy for what we are trying to maximize. Remember, the utilitarian answer is to do the most good per dollar spent, and, although it is plausible to think this happens when we tackle the ailments that rob of us of the most life years, this is an imperfect proxy. For example, if we are on the cusp of a major breakthrough for a rare disease (eg, chronic myelomonocytic leukemia), while still at the drawing board for a disease that is common (eg, pancreatic or lung cancer), investing in the former may make sense. For each dollar spent, we may in fact save more life years. Thus, we reach the conclusion that funding should be proportionate to efforts that are likely to bear fruit.

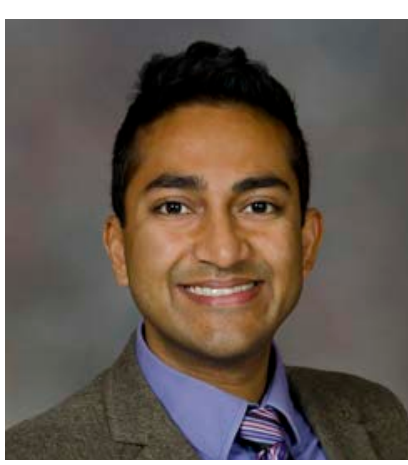

Vinay Prasad, MD, MPH

Vinay Prasad, MD, MPH, is a hematologist-oncologist and Assistant Professor of Medicine at the Oregon Health and Sciences University. He also holds appointments in the Division of Public Health and Preventive Medicine, and as a Senior Scholar in the Center for Health Care Ethics. He is nationally known for his research on oncology drugs, health policy, evidence-based medicine, bias, public health, preventive medicine, and medical reversal. Clinically, Dr. Prasad specializes in the care of lymphoma patients.

Dr. Prasad is a graduate of the University of Chicago Pritzker School of Medicine. He is also a graduate of Johns Hopkins Bloomberg School of Public Health and completed his undergraduate at Michigan State University. Dr. Prasad trained in general internal medicine at Northwestern University in Chicago, and completed his fellowship in Hematology and Oncology in the joint program between the $\mathrm{NCl}$ and National Heart, Lung and Blood Institute, both at the NIH.

Dr. Prasad is the author of more than 105 peer-reviewed articles in many journals, including the New England Journal of Medicine, and the Journal of the American Medical Association.

The ideas and viewpoints expressed in this commentary are those of the author and do not necessarily represent any policy, position, or program of NCCN. 
Prasad

At this point, the key difficulty in the question becomes apparent: how can we know which avenues of research will deliver big gains? Perhaps the difficulty in answering this question explains the enthusiasm for seizing upon one of the surrogates. Indeed, there remain few large-scale empirical analyses assessing and ranking the promise of research to translate to clinical success. One recent attempt studied patterns of failure among phase III oncology clinical trials, and concluded that poor activity in early phase trials was one adverse marker predicting against success. ${ }^{8}$ Whether the use of biomarker enrichment, or phase II randomization, or many other design features of clinical research seeking to improve reproducibility, will improve translation success warrants further exploration. But the short answer is that although is not clear what portfolio will result in the greatest good, believing that funding based on burden of disease is guaranteed to do so is also mistaken.

\section{Conclusions}

It is highly likely that the current funding system is suboptimal. It may also be likely that cancer groups that are the most vocal and well organized, and lack societal stigma (eg, non-smoking-related cancers), may generate disproportionate and perhaps unjustified funding. However, the common argument that research funding should be proportionate to causes of death or years of life lost is incorrect. What kills us and what robs of us of life simply approximate where progress is most likely to be achieved. They remain surrogates or stand-ins of where we may derive the most benefit. Appreciation of this fact may lead to more constructive debates about equitable cancer research funding and donation by tumor type.

\section{References}

1. Why Do Some Forms of Cancer Receive More Research Funding Than Others? Available at: http://www. independent.co.uk/life-style/health-and-families/health-news/why-do-some-forms-of-cancer-receive-moreresearch-funding-than-others-9771396.html. Accessed December 24, 2015.

2. The Truth About the Ice Bucket Challenge: Viral Memes Shouldn't Dictate Our Charitable Giving. Available at: http://www.vox.com/2014/8/20/6040435/als-ice-bucket-challenge-and-why-we-give-to-charity-donate. Accessed December 24, 2015

3. Cancer Funding: Does It Add Up? Available at: http://well.blogs.nytimes.com/2008/03/06/cancer-funding-doesit-add-up/. Accessed December 24, 2015.

4. Cancer Research: should investments reflect disease burden? Available at: http://healthydebate.ca/2012/10/topic/ wait-times-access-to-care/cancer. Accessed December 24, 2015.

5. Carter AJ, Nguyen CN. A comparison of cancer burden and research spending reveals discrepancies in the distribution of research funding. BMC Public Health 2012;12:526.

6. Gross CP, Anderson GF, Powe NR. The relation between funding by the national institutes of health and the burden of disease. N Engl J Med 1999;340:1881-1887.

7. NIH. Person Years of Life Lost. Available at: http://progressreport.cancer.gov/end/life_lost. Accessed December $24,2015$.

8. Seruga B, Ocana A, Amir E, Tannock IF. Failures in phase III: causes and consequences. Clin Cancer Res 2015;21:4552-4560. 